\title{
Circulating tumour DNA, microRNA and metabolites in cerebrospinal fluid as biomarkers for central nervous system malignancies
}

\author{
Soheil Zorofchian, ${ }^{1}$ Fatima Iqbal, ${ }^{1}$ Mayank Rao, ${ }^{2}$ Phyu P Aung, ${ }^{3}$ Yoshua Esquenazi, ${ }^{2}$ \\ Leomar Y Ballester ${ }^{1,2}$
}

'Department of Pathology and Laboratory Medicine, McGovern Medical School, The University of Texas Health Science Center at Houston, Houston, Texas, USA ${ }^{2}$ Vivian L. Smith Department of Neurosurgery, McGovern Medical School, The University of Texas Health Science Center at Houston, Houston, Texas, USA ${ }^{3}$ Department of Pathology, The University of Texas MD Anderson Cancer Center, Houston, Texas, USA

\section{Correspondence to} Dr Leomar Y Ballester, Department of Pathology and Laboratory Medicine, McGovern Medical School, The University of Texas Health Science Center at Houston, Houston, TX 77030 USA; leomar.y.ballester@ uth.tmc.edu and Dr Yoshua Esquenazi, Vivian L. Smith Department of Neurosurgery, McGovern Medical School, The University of Texas Health Science Center at Houston, Houston, TX, United States; Yoshua.EsquenaziLevy@uth. tmc.edu

Received 1 August 2018 Revised 11 October 2018 Accepted 21 October 2018 Published Online First 22 November 2018

D Check for updates

(c) Author(s) (or their employer(s)) 2019. No commercial re-use. See rights and permissions. Published by BMJ.

To cite: Zorofchian $\mathrm{S}$, lqbal F, Rao M, et al. J Clin Pathol 2019:72:271-280

\section{ABSTRACT}

Central nervous system (CNS) malignancies can be difficult to diagnose and many do not respond satisfactorily to existing therapies. Monitoring patients with CNS malignancies for treatment response and tumour recurrence can be challenging because of the difficulty and risks of brain biopsies, and the low specificity and sensitivity of the less invasive methodologies that are currently available. Uncertainty about tumour diagnosis or whether a tumour has responded to treatment or has recurred can cause delays in therapeutic decisions that can impact patient outcome. Therefore, there is an urgent need to develop and validate reliable and minimally invasive biomarkers for CNS tumours that can be used alone or in combination with current clinical practices. Blood-based biomarkers can be informative in the diagnosis and monitoring of various types of cancer. However, bloodbased biomarkers have proven suboptimal for analysis of CNS tumours. In contrast, circulating biomarkers in cerebrospinal fluid (CSF), including circulating tumour DNA, microRNAs and metabolites, hold promise for accurate and minimally invasive assessment of CNS tumours. This review summarises the current understanding of these three types of CSF biomarkers and their potential use in neuro-oncologic clinical practice.

\section{INTRODUCTION}

Central nervous system (CNS) tumours are a major health problem and surpass leukaemia as the leading cause of cancer death among children and adolescents. ${ }^{1}$ CNS tumours are associated with high rates of mortality and morbidity. ${ }^{12}$ The absence of effective therapies is a challenge in neuro-oncology. Moreover, current methods for diagnosing and monitoring CNS tumours, such as cerebrospinal fluid (CSF) cytology and MRI, have low sensitivity and specificity, respectively. MRI often fails to discriminate neoplastic from non-neoplastic conditions. Furthermore, serial MRI and CT are suboptimal methods to detect changes associated with treatment response or disease progression. ${ }^{3}$ In these situations, treatment decisions are often postponed until imaging studies definitely clarify tumour behaviour, while patients miss potential therapeutic opportunities that could impact survival.

CSF cytology is currently considered the gold standard for the diagnosis of leptomeningeal disease $(\mathrm{LMD})^{4}$; however, CSF cytology has some critical drawbacks. First, it is a qualitative assay based on microscopic examination with high interobserver variability. ${ }^{5} 6$ Second, despite acceptable specificity, CSF cytology suffers from low sensitivity and false-negative results are common. ${ }^{7-9}$ Third, CSF cytology requires the presence of morphologically intact tumour cells to be examined under the microscope, which contributes to its low sensitivity. ${ }^{10}$ Analysing circulating biomarkers derived from tumour cells, including circulating tumour DNA (ctDNA), miRNA and metabolites, has the potential to circumvent some of the limitations of CSF cytology (figure 1).

\section{Rationale for development of CSF biomarkers for CNS tumours}

Although CNS malignancies are generally associated with a dreary prognosis and poor quality of life, studies in recent years have led to promising therapeutic advances. ${ }^{2} 11$ These advances have the potential to shift treatment approaches from diseasetype-based therapy to individual-genotype-based therapy, which relies on knowledge about the genomic alterations in the tumour. ${ }^{12}$ Currently, genomic characterisation of CNS tumours requires tissue obtained via surgical procedure. Furthermore, because of temporal and spatial intratumour heterogeneity, determining the genomic alterations of CNS tumours may require repeated biopsies. ${ }^{13} 14$ Some tumours are not amenable to surgical intervention because they are in critical anatomical regions (eg, the brainstem) or because comorbidities make the patient unable to tolerate a surgical procedure. In addition, neither of the current methods for diagnosing and monitoring CNS tumours (ie, MRI and CSF cytology) offers information about tumour-associated mutations. ${ }^{4}$

Blood is one of the easiest biofluids to collect, and circulating biomarkers in blood can be useful in the diagnosis and monitoring of patients with cancer. ${ }^{1516}$ It has been demonstrated that circulating biomarkers in blood can be useful in the diagnosis and monitoring of patients with tumours that occur outside the CNS. As a non-invasive screening tool with minimal risk, plasma analysis was found to be informative in cancer detection, mutation profiling, evaluation of response to therapy and tumour recurrence, and detection of emergent resistance to ongoing therapies in various cancers, including prostate, breast and lung. ${ }^{17-21}$ Detection of ctDNA in blood samples without measurable circulating 

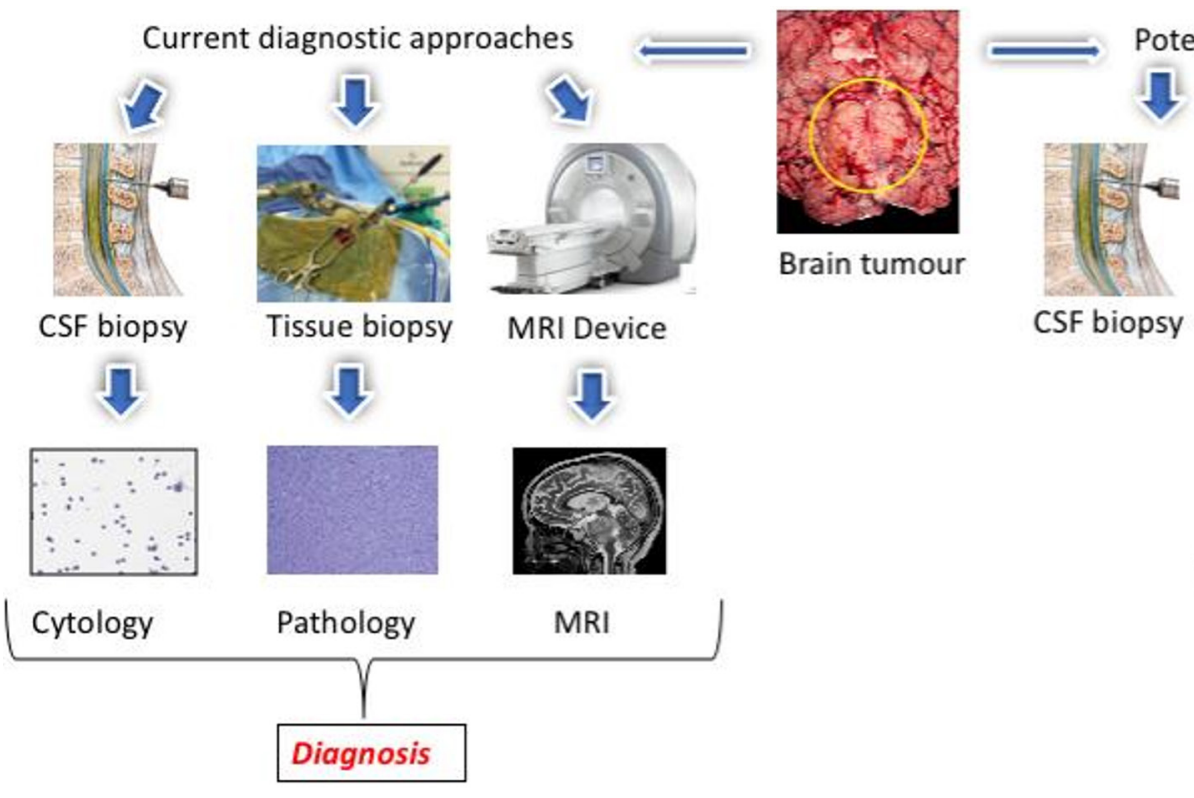

Potential diagnostic approaches
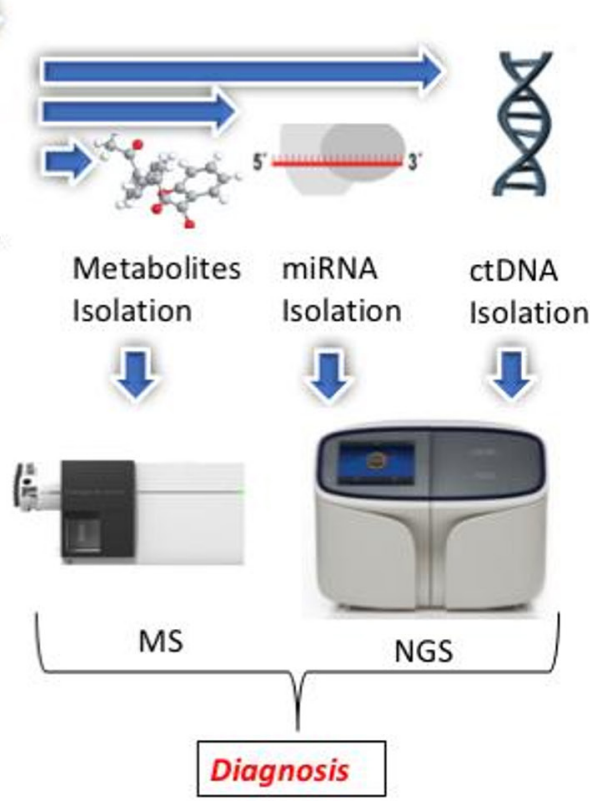

Figure 1 Comparison of current and possible future CSF biomarker-based approaches to diagnosis and monitoring of patients with central nervous system (CNS) malignancies. Left: MRI, tissue pathology and CSF cytology are current approaches that are routinely used for the CNS tumour diagnosis. Right: cancer cells shed or secrete different biomolecules into the CSF circulation, including ctDNA, miRNA and metabolites. Isolation and analysis of these biomarkers from CSF can provide critical real-time information regarding the tumour genome and microenvironment. CSF, cerebrospinal fluid; ctDNA, circulating tumour DNA; NGS, next-generation sequencing.

tumour cells suggests that ctDNA in plasma can provide useful information about tumours independent of the presence of circulating tumour cells. ${ }^{22}$

However, because of the presence of the blood-brain barrier, blood is not an ideal fluid to accurately evaluate biomarkers derived from CNS tumours. ${ }^{1112}$ Moreover, blood is not a suitable candidate to evaluate metastatic CNS tumours if the patients have coexisting systemic tumours (ie, non-CNS disease). ${ }^{23} \mathrm{~A}$ recent study on blood samples collected from 640 patients with various cancer types suggested that ctDNA is a promising biomarker for solid tumours outside the CNS, including bladder, breast, colorectal, gastro-oesophageal, hepatocellular, ovarian, pancreatic, and head and neck cancer and melanoma. However, ctDNA was detected in the blood of fewer than $10 \%$ of patients with gliomas. ${ }^{22}$ Several studies also reported the sensitivity for circulating tumour cells in the blood of patients with glioblastoma as low as $21 \%$ to $39 \% .{ }^{24-27}$ These studies strongly suggested that the blood-brain barrier can be an obstacle that prevents circulating tumour cells or ctDNA from entering the blood.

In contrast to blood, CSF is in direct contact with the CNS and is a suitable source of biomarkers from CNS tumours. ${ }^{3-7}{ }^{12-14} \mathrm{~A}$ number of tumour-derived biomarkers have been demonstrated in the CSF of patients with CNS tumours, including ctDNA, miRNA and metabolites. ${ }^{4-8} 1214$ Furthermore, CSF is easier and safer to obtain than CNS tumour tissue. However, at present, CSF biomarker analysis is rarely used in the care of patients with CNS tumours because of the lack of well-established biomarkers with clinical use. In addition, performing lumbar puncture for CSF sampling is categorised as minimally invasive and is frequently performed as part of clinical care for patients with different CNS tumours, such as lymphomas and medulloblastoma. ${ }^{28}{ }^{29}$ In this review, we discuss recent work evaluating three types of tumour-derived biomarkers that can be detected in the CSF (ctDNA, miRNA and metabolites) and their potential implementation in the clinical setting.

\section{Potential benefits of CSF biomarkers for patients with CNS tumours}

Areas in which CSF biomarkers may improve current clinical practice are (1) early diagnosis before surgery, (2) diagnosis of tumours that are not amenable or typically treated by surgery, (3) early identification of tumour recurrence, (4) monitoring of response to treatment and (5) facilitating use of precision-medicine therapies.

\section{Diagnosis}

One of the principal determinants of the clinical management of primary or metastatic CNS tumours is the tumour type. Tumour type is usually determined from tissue-based studies performed after surgical intervention, and subsequent decisions about surveillance, chemotherapy and radiotherapy are influenced by the tissue-based diagnosis. Even in patients with CNS diseases for which surgical management is not indicated (eg, CNS lymphoma, midline gliomas), a brain biopsy is currently required to establish a diagnosis and plan further patient care. The ability to distinguish lymphoma from diffuse glioma by analysing CSF biomarkers could avoid the need for a brain biopsy in some patients. In addition, in the current era of molecular classification of CNS tumours, it is conceivable that preoperative knowledge of biomarkers with prognostic significance might help in surgical planning, intraoperative decision-making and clinical trial enrolment.

\section{Identification of tumour recurrence}

Post-treatment monitoring of patients with primary or metastatic CNS tumours is challenging because alterations due to 
secondary effects of chemoradiation or pseudoprogression (eg, radiation necrosis) cannot be reliably distinguished from tumour recurrence on imaging. ${ }^{30} 31$ Because of our inability to distinguish 'pseudoprogression' from recurrence, ${ }^{32}$ some patients without true tumour recurrence are subjected to potentially unnecessary surgical procedures such as re-resections or biopsies to confirm recurrence, which can have a high rate of sampling error. It has been suggested that the levels of tumour biomarkers (ctDNA, miRNA and metabolites) in CSF might correlate with CNS tumour burden. In this scenario, it is reasonable to hypothesise that tumour progression will lead to an increase in the levels of tumour-derived biomarkers in CSF, which will facilitate distinguishing progression from pseudoprogression. The ability to reliably distinguish progression from pseudoprogression may reduce unnecessary surgical procedures in patients with imaging alterations due to treatment effect.

\section{Monitoring treatment response}

Imaging studies (conventional MRI, perfusion MRI and CT, MR spectroscopy, PET) often fail to provide conclusive evidence to distinguish tumour progression from treatment response. Other biomarkers have been investigated for monitoring treatment response of CNS tumours, ${ }^{334}$ but none have clinically proved sensitive and specific. The absence of sensitive and specific biomarkers to evaluate treatment response in patients with CNS tumours hampers the development of new therapeutics in neuro-oncology. Future studies should aim to close this knowledge gap by systematically evaluating CSF samples for circulating biomarkers (ctDNA, miRNA and metabolites) and correlating the levels of tumour-derived biomarkers with tumour volume assessed by MRI.

\section{ctDNA in CSF}

Previously, molecular techniques such as digital droplet PCR (ddPCR) and BEAMing were implemented to detect specific mutations in biofluids. ${ }^{35}$ However, next-generation sequencing (NGS) provides an opportunity to screen for novel mutations and even gene fusions and amplifications with a limit of detection below $1 \% .^{36}$ In addition, commercially available NGS devices can conduct CSF genomic analysis at a reasonable cost. Although the rate of ctDNA detection in CSF is not 100\%, the rate of ctDNA detection in CSF appears relatively similar to that of other biofluids. ${ }^{37}$ Also, the rate of ctDNA detection in CSF is higher than the rate of detection of intact tumour cells by microscopic examination. ${ }^{38} 39$

Two main approaches have been used to analyse ctDNA in CSF: single-gene analysis and multigene panel sequencing. Single-gene analysis is most suitable for when genetic alterations present in the tumour are known, or tumours for which hot-spot mutations have been thoroughly characterised and can be assessed in CSF. ${ }^{23}$

In contrast, multigene sequencing by NGS allows the interrogation of multiple genes, and it is the best approach for when there is no knowledge about the specific mutations present in a tumour. CSF ctDNA can be comprehensively examined for mutations in multiple known cancer-associated genes. All types of genetic alterations including point mutations, gene fusions, gene copy number alterations and small insertions/deletions can be detected in CSF. ${ }^{23} 384041$ However, the clinical sensitivity of this approach remains to be determined in well-controlled studies with CSF samples obtained at the time of presentation, before the patients are treated.

\section{Single-gene assessment of CSF ctDNA}

One of the first studies analysing CSF ctDNA for alterations in a single gene was performed by Rhodes et al in the 1990s. They reported detection of mutations in the CSF of two patients with intracranial neoplasms. One patient had metastatic breast cancer with amplification of the ERBB2 gene, and the other had glioblastoma with amplification of EGFR. These amplifications were detected in CSF by PCR. ${ }^{31}$ The same group reported detection of TP53 mutations in the CSF of a patient with glioblastoma. ${ }^{32}$

Following these promising results, various groups have examined CSF ctDNA in patients with CNS tumours and have shown that CSF ctDNA is superior to CSF cytology. For example, in patients with LMD, the current gold standard for diagnosis is microscopic examination of the CSF for the presence of intact tumour cells (ie, CSF cytology). Although CSF cytology is relatively specific for LMD, it has a low sensitivity. ${ }^{39}{ }^{42}$ Therefore, better assays for diagnosing and monitoring patients with LMD are urgently needed. Rhodes et al examined 31 CSF samples from 21 patients for the presence of tumour cells by CSF cytology and for immunoglobulin gene rearrangement by PCR. ${ }^{43}$ PCR examination of the CSF yielded positive results even in specimens for which conventional CSF-cytology analysis was negative. Shingyoji et al studied 29 patients with lung adenocarcinoma for EGFR mutations in the CSF. ${ }^{44}$ Intriguingly, $31 \%$ of EGFR mutations in CSF ctDNA were detected in patients with negative CSF-cytology results. Similarly, in another study, KRAS mutations were detected in the CSF of two patients with lung adenocarcinoma who had negative CSF-cytology results. ${ }^{45}$ In addition to having a role in diagnosis, CSF-ctDNA analysis may have a role in the evaluation of tumour progression. In a recent study, the levels of the BRAF p.V600E mutation in CSF from a patient with metastatic melanoma and LMD appeared to correlate with treatment response and tumour recurrence. ${ }^{46}$ A correlation between mutation levels analysed by ddPCR and tumour size has been confirmed in a study of 12 patients with primary and metastatic CNS tumours. ${ }^{27}$

In CNS lymphoma, detection of MYD88 p.L265P was suggested to be a promising candidate for diagnosis due to the high specificity and the prevalence of this mutation. ${ }^{47} \mathrm{~A}$ recent study detected MYD88 p.L265P mutation in the CSF of $1 / 3$ patients with primary CNS lymphoma. ${ }^{48}$

Diffuse midline glioma, H3-K27M-mutant (formerly known as diffuse intrinsic pontine glioma) are paediatric high-grade gliomas with less than $20 \%$ survival at 2 years. Because of the tumour's anatomical location, surgical resection is not feasible and tissue biopsies are challenging. ${ }^{41}{ }^{49}$ Therefore, development of a minimally invasive diagnostic assay will be of great clinical use. A recent study attempted to take advantage of the high rate of histone $\mathrm{H} 3$ mutations (p.K27M) in patients with diffuse midline gliomas for detection of CSF ctDNA using either H3F3A c.83 A > T detection via nested PCR or targeted Sanger sequencing. CSF analysis of 11 children with these tumours showed successful detection of histone mutations with $87.5 \%$ sensitivity and $100 \%$ specificity. ${ }^{40}$

\section{Multigene assessment of CSF ctDNA}

The use of multigene cancer panels for analysis of CSF ctDNA has been the focus of several recent studies. ${ }^{38-4150}$ Using this approach, CSF of a patient with suspected leptomeningeal metastasis from lung adenocarcinoma was analysed for mutations in 578 cancer genes. Seven somatic mutations in CSF ctDNA were identified, in agreement with the results of sequencing analysis from the primary tumour biopsy specimen. ${ }^{23}$ Pentsova 
et al analysed CSF ctDNA from 53 patients for alterations in 341 cancer-associated genes by NGS. Somatic alterations were detected in 6/12 (50\%) patients with primary brain tumours, 20/32 (63\%) patients with metastatic solid tumours and 0/9 patients without a CNS tumour. ${ }^{38}$

\section{Possible limitations of CSF-ctDNA analysis}

While CSF-ctDNA analysis is a promising tool for the evaluation of patients with CNS tumours, studies have shown that CSF ctDNA cannot be detected in every patient with a CNS malignancy. Factors that determine the success of CSF-ctDNA analysis are unclear, although it has been postulated that the tumour's location within the brain, and its proximity to the CSF, might be a contributing factor. ${ }^{37}$ In a study of 35 patients with primary CNS tumours, ctDNA was detected in 74\% of the CSF samples. ${ }^{37}$ In this study, all tumours with at least one somatic mutation were identified, and then CSF samples were examined for the same DNA mutations using targeted or genome-wide sequencing. The CSF of patients whose tumours were not directly exposed to the CSF had no detectable ctDNA, but ctDNA was detected in samples collected from all ependymomas, medulloblastomas and high-grade gliomas abutting the ventricles. The technical sensitivity of the methodology used to detect mutations in CSF ctDNA is also a critical contributing factor. Pan et al found different concentrations of tumour-derived mutant alleles in CSF samples from 6/7 patients with solid brain tumours using ddPCR and targeted amplicon sequencing. ${ }^{23}$ In contrast, one study using ddPCR failed to detect ctDNA in CSF collected from three patients with intramedullary spinal ependymomas which were in direct contact with the CSF. ${ }^{49}$

\section{miRNAs in CSF}

MiRNAs, discovered in the 1990s, are small regulatory non-coding RNA fragments that regulate gene expression at a post-transcriptional level by degrading messenger RNAs or directly interfering with translation initiation. ${ }^{51}$ There is increasing evidence that miRNAs can function as oncogenes (oncomirs) and/or tumour suppressors, and that dysfunctional expression of miRNAs is a common feature of many types of cancer. Various studies have shown linkage of miRNAs with CNS malignancies, such as medulloblastoma, B-cell lymphoma and glioblastoma. ${ }^{51-55}$ The existence of the blood-brain barrier has led to the hypothesis that miRNAs in the CSF would reflect brain's physiology and pathological processes more accurately than miRNAs in other body fluids. ${ }^{53}$ This supports the analysis of CSF miRNAs as biomarkers for CNS tumours. Therefore, several studies have investigated the role of miRNA expression analysis in the diagnosis of CNS malignancies and their potential prognostic significance. However, studies of CSF miRNAs are limited. ${ }^{51-55}$

Brain miRNA profiles may differ depending on the region of brain being studied. ${ }^{52}$ An association between miRNAs and brain cancer was discovered in 2005, and miRNAs in circulating body fluids from patients with brain cancer were first detected in 2008. ${ }^{54}$ Multiple miRNAs in CSF have been found to be significantly associated with primary and metastatic CNS tumours. Table 1 shows different miRNAs and their association with various CNS malignancies. It has been observed that the same miRNA can be upregulated in one CNS malignancy and downregulated in another, indicating that combinations of miRNA signatures may be useful in distinguishing different CNS tumour types. $^{56}$

Table 1 Summary of microRNAs studied in CSF samples of patients with CNS tumours

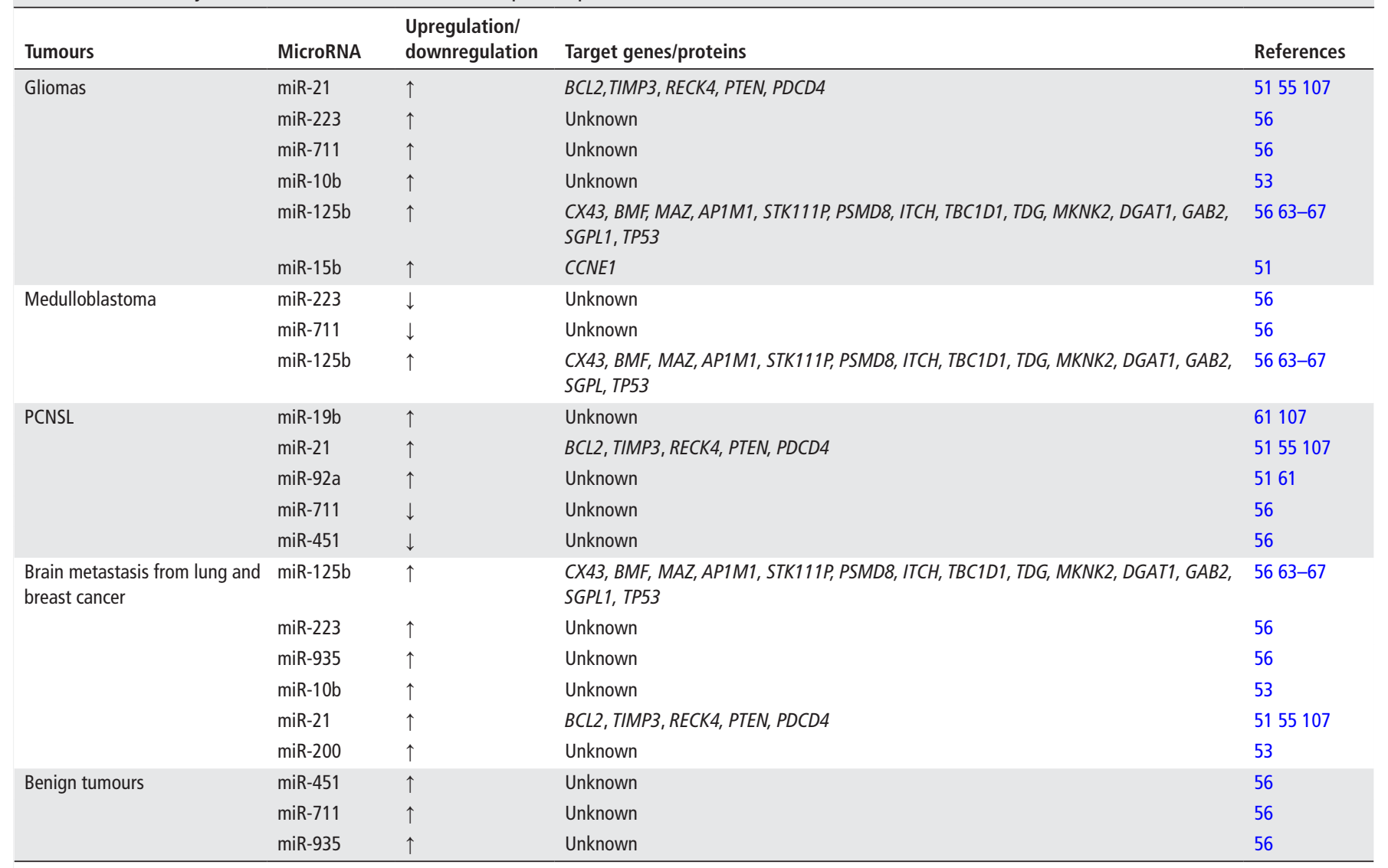

CNA, central nervous system; CSF, cerebrospinal fluid; PCNSL, primary central nervous system lymphoma. 
The majority of extracellular miRNAs circulating in biofluids are in extracellular vesicles (EVs), such as apoptotic bodies, microvesicles and exosomes. Exosomes are extracellular vesicles that contain a variety of biological molecules and have been shown to be enriched with tumour-derived miRNAs. ${ }^{57} 58$ Although the exact origins of miRNAs found in CSF samples is unclear, miRNAs in EVs are considered highly stable and resistant to degradation, which makes them ideal candidates for diagnostic biomarkers. Evidence suggests that incorporation of miRNAs into EVs results in protection from degradation in biofluids. ${ }^{59}$ The feasibility of isolating and sequencing exosomal miRNA from CSF has been demonstrated. ${ }^{51-55}$

\section{Detection of miRNAs in CSF}

Currently, there are four major approaches for detecting miRNAs in CSF: qRT-PCR, DNA microarrays, in situ hybridisation and RNA sequencing. ${ }^{54}$ The qRT-PCR method is widely used since it is quantitative, extremely sensitive, highly specific and reproducible. After CSF is collected, miRNAs can be isolated and analysed. CSF is collected via lumbar puncture, which is a minimally invasive procedure. However, it may cause complications such as infection, bleeding, headache, back pain and cerebral herniation. ${ }^{54}$ To prepare a cell-free miRNA sample, minimising contamination of CSF at the time of collection is a crucial step, along with CSF preservation and storage.

\section{Specific miRNAs of interest}

MiR-21

MiR-21 is the most commonly overexpressed miRNA in gliomas and primary central nervous system lymphoma (PCNSL). ${ }^{5153-556061}$ Levels of exosome-derived miR-21 significantly correlate with recurrent glioma. ${ }^{55}$ Similarly, miR-21 is overexpressed in the CSF of patients with PCNSL. Moreover, miR-21 levels in the CSF significantly correlated with worst prognosis among patients with diffuse B-cell lymphoma. ${ }^{5154} 61$ MiR-21 affects major cancer genes, such as BCL2, RECK4, phosphate and tensin homologue (PTEN), and PDCD4. ${ }^{51}{ }^{55}$ MiR-21 also influences the mitochondrial-initiated apoptosis pathways and promotes tissue invasion by targeting and inhibiting the TIMP3 protein. ${ }^{51}$ Because so much research has been done on miR-21, it has been established that miR-21 is one of the most important clinical CNS biomarkers in CNS malignancies.

\section{MiR-10b and miR-200}

Under normal conditions, miR-10b can be detected in blood but not in CSF; the presence of miR-10b in CSF is a potentially unique biomarker for CNS metastatic tumours. ${ }^{53}$ However, miR-10b was also found to be significantly expressed in glioblastoma tissues in different studies. MiR-10b has been shown to be overexpressed in the CSF of patients with brain and leptomeningeal metastases from lung and breast cancer. MiR-200 family (miR-200a, miR-200b, miR-200c and miR-141), unlike miR-10b, is not seen in glioblastoma tumours but is found in abundant quantities in brain metastases from primary lung and breast cancers. This makes miR-200 a candidate biomarker to distinguish glioblastoma from metastatic lung or breast cancer to the CNS. ${ }^{53}$

\section{$\operatorname{MiR}-15 b$}

MiR-15b is a member of the miR-15/16 family and its level is significantly elevated in gliomas. Xia et al suggested miR-15b plays an important role in glioma genesis by regulating cell-cycle progression and functioning as a tumour suppressor gene. ${ }^{5162}$
MiR-15b targets cyclin E1 (CCNE1), which inhibits cell proliferation by arresting the cell cycle at G1 phase. Baraniskin et al reported that a combination of miR-15b and miR-21 would have better diagnostic value for glioma tumours than the levels of miR-21 alone. ${ }^{51}$

\section{MiR-19b and miR-92a}

MiR-19b and miR-92a can be helpful in the diagnosis and monitoring of PCNSL. After miR-21, these miRNAs are the second and third most highly expressed miRNAs in such patients. Both miR-19b and miR-92a are members of the poly-cistronic miR-17-92 cluster located on human chromosome $13 .{ }^{61}$

\section{MiR-451, miR-711 and miR-935}

Drusco et al investigated the miRNA profile in 48 CSF samples from 6 different groups of patients: normal, benign, glioblastoma, medulloblastoma, metastasis and CNS lymphoma. In patients with a CNS tumour, simultaneous testing for miR-451, miR-711 and miR-935 could help establish a signature profile to diagnose and differentiate various neoplastic lesions. Normal group was shown to moderately express miR-711 and miR-935 with the lowest expression of miR-451 compared with pathological groups. Moderate expression of all three miRNAs indicates a benign CNS tumour. Absence of miR-935 expression with low expression of miR-451 and extremely low expression of miR-711 may indicate the presence of a CNS lymphoma. Absence of miR-935 expression with moderate expression of miR-451 and miR-711 was strongly correlated to glioblastoma or medulloblastoma. ${ }^{56}$

\section{MiR-125b and miR-223}

Both miR-125b and miR-223 are important diagnostic biomarkers for glioblastoma, medulloblastoma and brain metastasis. In the study by Drusco et al, expression of these miRNAs, along with expression of miR-711, was helpful in differentiating glioblastoma from medulloblastoma. In comparison to patients with medulloblastoma, patients with glioblastoma exhibit increased expression of miR-223 and miR-711 and a decreased expression of miR-125b. Conversely, patients with medulloblastoma exhibit a decreased expression of miR-223 and miR-711 and an increased expression of miR-125b. The CSF of patients with brain metastases from primary breast and lung tumours exhibits variable elevated expression of miR-125b and miR-223 along with high expression of miR-935..$^{56}$

MiR-125b is one of the most abundant miRNAs in the brain. It promotes neuronal differentiation by repressing multiple target genes, such as AP1M1, STK111P, PSMD8, ITCH, TBC1D1, TDG, MKNK2, DGAT1, GAB2 and SGPL1. ${ }^{63} \mathrm{MiR}-125 \mathrm{~b}$ has been shown to have both tumour-promoting and tumour-suppressing effects in CNS cancer. Several studies have indicated that miR-125b promotes proliferation and growth of glioma cells by targeting the cell apoptosis-related protein Bcl-2 modifying factor, inhibiting connexin-43 expression, and negatively regulating $P 53$ gene expression and p53-induced apoptosis. ${ }^{64-66}$ Other studies have shown that miR-125b acts as a tumour suppressor in glioblastoma-associated endothelial cells and glioma stem cell proliferation. ${ }^{67}{ }^{68} \mathrm{MiR}-125 \mathrm{~b}$ regulates Myc-associated zinc finger protein (MAZ) and mediates VEGF-induced angiogenesis in Glioblastoma. ${ }^{67}$

Increased miR-223 expression promotes tumour growth and invasion in glioblastoma cell lines by targeting PAX6 and suppressing glial precursor proliferation through inactivation of NFIA (nuclear factor IA). ${ }^{56}$ 


\section{Potential applications of CSF miRNA detection and profiling}

The results of various studies suggest that levels of miRNAs in the CSF can help distinguish between CNS tumours and other inflammatory, degenerative and traumatic CNS anomalies. $^{51545660}$ The use of CSF miRNAs as biomarkers can be extremely helpful in the management of patients with CNS malignancies that are not suitable for surgical management. ${ }^{53}$ Data suggest that a diagnostic assay could be established on the basis of the CSF miRNA profile of different CNS diseases. MiRNA profiling could potentially also be used for evaluating relapse, post-treatment follow-up, and examination of the efficacy of chemotherapy and radiotherapy. ${ }^{53}$

\section{Future directions}

Evidence of the role of miRNAs in numerous neurological diseases, mapping of the expression of new miRNAs, identification of miRNA target genes/proteins and the gene/protein's associated pathways with neurological diseases all lead to high optimism regarding the use of miRNAs as effective diagnostic and prognostic biomarkers for CNS diseases. Using miRNAs as biomarkers for CNS diseases will be ideal when miRNAs are easy to extract, sequencing is less expensive and miRNA assays are sensitive enough for quantitative analysis with low sample volumes. Despite the limitations noted above, miRNA-based biomarkers continue to be an exciting area of study in medicine.

\section{Limitations}

Despite advances in miRNA isolation, storage and sequencing technology, the use of miRNAs as biomarkers has some limitations. These include inadequate CSF sample collection, improper extraction methods, lack of proper protocols for sample handling and storage, sample contamination and lack of standardised isolation procedures for use in the clinical setting. Limited knowledge about environmental factors that may affect miRNA expression in patients may also limit the clinical use of this approach.

\section{METABOLOMICS}

Metabolites are small molecules that are intermediates or products of biochemical reactions such as glycolysis or synthesis of cellular macromolecules. Metabolites can be measured both in vitro and in vivo in cells, fluids or tissues using nuclear magnetic resonance or mass spectrometry-based approaches, including liquid chromatography/mass spectrometry and gas chromatography/mass spectrometry. ${ }^{6970}$ Nuclear magnetic resonance is a rapid, non-destructive and reproducible technique that requires minimal sample preparation and offers highly essential information but has a low sensitivity. Mass spectrometry, in contrast, is highly sensitive and has a wide dynamic range. ${ }^{23} 7071$ Recent developments in nuclear magnetic resonance and mass spectrometry analyses have improved the sensitivity and resolution of available assays. As a result, hundreds to thousands of small molecules can be identified and quantified from a small biological sample.

Methods that can be used to study small metabolites in biological samples are untargeted metabolomics, metabolic profiling ('targeted metabolomics') and stable isotope-resolved metabolomics. Untargeted metabolomics refers to the study of all detectable metabolites from a sample. Targeted metabolomics is commonly used to identify and quantify a predefined list of metabolites from a sample. In stable isotope-resolved metabolomics, stable isotope-assisted metabolomics is used to calculate intracellular and extracellular metabolic changes. These stable isotopes include carbon (carbon 13), nitrogen (nitrogen 15), deuterium and oxygen (oxygen 18). ${ }^{7273}$ For clinical applications, metabolomic analysis has some advantages over genomics: for example, the measurement of metabolites is relatively cheap on a per-sample basis, is fully automated and the metabolome of one sample can easily be compared with the metabolome of another. ${ }^{74}$

Cancer cells have altered cellular metabolism, and mutations in genes associated with key metabolic pathways (eg, IDH1/IDH2) are important drivers of CNS tumours. ${ }^{22} 23$ For example, the production of abnormal oncogenic metabolites (eg, D-2-hydroxyglutarate) has been documented in CNS tumours that carry IDH1 or IDH2 mutations. ${ }^{24}{ }^{25}$ Moreover, alterations in other CSF metabolites (eg, elevations in aminoadipic acid, acetylcarnitine and shikimate) in patients with CNS tumours have been demonstrated. ${ }^{313}$

\section{Metabolites in CSF and CNS tumours}

Tumour cells need significant amounts of energy to support their aberrant growth, and they obtain this energy by altering cellular metabolic processes, such as glycolysis, the tricarboxylic acid (TCA) cycle, amino acid metabolism and lipid metabolism. ${ }^{75}$ With the use of metabolomics, metabolic perturbations in cancer cells versus normal cells have been identified in colon, leukaemia, ovarian, oesophageal, oral and renal cancers. ${ }^{76-80}$ Abnormal metabolites are involved in several metabolic pathways like glycolysis, TCA cycle, pentose phosphate pathway, fatty acid metabolism and amino acid metabolism. ${ }^{70} 81$

Currently, the largest publicly available database of metabolites is the Human Metabolome Database (HMDB), which Wishart et al described in a 2018 review paper. ${ }^{82}$ This database contains 1 14000 known human water-soluble and lipid-soluble metabolites. Once produced, they can be measured in tissue as well as biological fluids like blood, plasma, CSF, urine and sweat. Abnormal levels of metabolites might suggest the existence of a disease process. ${ }^{70}$ At the time of this review, the HMDB lists 450 metabolites that have been identified in CSF.

Cellular metabolites are altered in infective, inflammatory and neoplastic brain tissues and are secreted in CSF. Measurement of these metabolites in CSF can help in early diagnosis and prognostication of patients with various brain conditions. ${ }^{78183}$ Here, we discuss the metabolites that have been found to be altered in the CSF of patients with CNS tumours.

\section{Glycolysis and TCA cycle metabolites}

Under aerobic conditions, human cells use oxidative phosphorylation as the main source of ATP production. It has been reported that tumour cells use glycolysis as their main source of energy, even under aerobic conditions. This phenomenon is called the Warburg effect as it was first described by the Nobel laureate Otto Heinrich Warburg. ${ }^{56} \mathrm{He}$ postulated that tumour cells use glucose for energy and transform it into lactate by the enzyme lactate dehydrogenase. ${ }^{81} 8485$ In this sense, tumour cells differ from normal cells, which transform glucose into pyruvate and then metabolise pyruvate in the mitochondria by oxidative phosphorylation. Hence, tumour cells rely more in aerobic glycolysis and lactic acid fermentation, than the TCA cycle, for ATP production. Also, tumours often exhibit necrosis and ischaemia, which further elevates lactate levels. ${ }^{86}$

Lactate can be measured in the CSF of patients with brain tumours. Chow et al retrospectively reviewed the lactate concentration in CSF of 2268 patients from 1992 to 2002. Only 159 patients had lactate levels of $2 \mathrm{mmol} / \mathrm{L}$ or greater, and $4 \%$ of 
them were diagnosed with brain or meningeal tumours. ${ }^{87}$ In a case report published in 2008, Blüher et al reported that in a 3 -year-old boy with malignant meningeal melanoma, measurement of $\mathrm{pH}$ in CSF showed central lactic acidosis despite alkalosis in peripheral blood. ${ }^{88}$ These authors hypothesised that lactate production by the tumour might induce central acidification. The enzymes that are involved in lactate production (eg, lactate dehydrogenase) increase in concert with increases in lactate levels during brain tumour proliferation. ${ }^{8185}$

Glucose and glutamine are the main substrates that tumour cells use to produce energy. Tumour cells convert glucose and glutamine into lactate by the enzyme lactate dehydrogenase. ${ }^{81} 8485$ Proliferating cells also use both glucose and glutamine for other purposes such as to enhance protein, nucleic acid and fatty acid synthesis. Glucose and glutamine provide acetyl-CoA and NADPH, which are needed to synthesise lipids. Hypoglycorrhachia, a decrease in glucose levels in the CSF, is associated with bacterial and fungal infections, but it also has been described in patients with brain tumours. ${ }^{89} 90$ The underlying causes might be increased anaerobic glycolysis, blood-brain-barrier blockage or defects in glucose transport to the CSF. Chow et al studied cases of hypoglycorrhachia at a tertiary teaching hospital over a period of 5 years and found that brain tumours were the underlying cause in $\sim 11 \%$ of cases. $^{87}$

\section{TCA cycle metabolites}

Metabolic alterations in the TCA cycle have also been reported along with alterations in glycolysis. The TCA cycle, or citric acid cycle, consists of several reactions that ultimately lead to production of energy in the form of ATP. There is oxidation of acetyl-CoA derived from carbohydrates, fats and proteins. In brain tumour cells, the cycle gets altered. Citrate and isocitrate levels have been shown to be elevated in the CSF of patients with glioblastomas. ${ }^{81}$ Glycolytic activity exceeds the TCA cycle activity and causes accumulation of citrate and lactate. The levels of these metabolites were significantly higher in IDH1/IDH2 mutant glioma cells than in cells with wild-type IDH1/IDH2 genes. ${ }^{91}$ IDH1 (isocitrate dehydrogenase 1 ) is an enzyme located in the cytosol and peroxisome and converts isocitrate to $\alpha$-ketoglutarate. IDH1 is frequently mutated in gliomas. ${ }^{92}$ IDH1/IDH2 mutations in gliomas facilitate conversion of $\alpha$-ketoglutarate to D-2-hydroxyglutarate (D-2-HG). ${ }^{93}$ Elevation in D-2-HG levels inhibits $\alpha$-ketoglutarate-dependent enzymes and causes hypermethylation of histones and DNA, which results in altered gene expression, resulting in activation of oncogenes and inactivation of tumour-suppressor genes. ${ }^{75} 76$ Thus, D-2-HG levels could be helpful in the diagnosis of malignant brain tumours. ${ }^{76}$ One study showed elevated D-2-HG levels in the CSF of patients with IDH-mutant gliomas. ${ }^{94}$ Also, citric acid and isocitric acid have been shown to be elevated in the CSF of patients with IDH-mutant gliomas. ${ }^{81}$ Locasale et al also found differences in the levels of various metabolites in the CSF of patients with gliomas compared with controls, including alterations in TCA cycle metabolites. ${ }^{83}$ However, further studies are needed to understand changes in metabolites that take place in the CSF of patients with CNS tumours and how this information could be used in the clinical setting.

\section{Myo-inositol}

Myo-inositol is a natural sugar found in cell membrane phospholipids and plasma lipoproteins. Myo-inositol is involved in cell signalling and may stimulate tumour cell differentiation. Using MR spectroscopy, Kallenberg et al and Castillo et al independently reported that myo-inositol levels are increased in patients with glioma compared with controls. ${ }^{95} 96$ However, Locasale et al failed to detect increased myo-inositol levels in CSF obtained from patients with gliomas compared with controls. ${ }^{83}$ In addition to myo-inositol, elevated level of cholesteryl sulfate in the CSF has been found in brain tumours, and cholesteryl sulfate level correlated with increased severity and proliferation of brain tumour cells. Cholesteryl sulfate is a component of the cell membrane, has a stabilising function on the membrane and is involved in signal transduction.

\section{Inflammatory markers and amino acids}

Brain tumours are frequently accompanied by ischaemia, necrosis and inflammation. The inflammatory markers can be measured in the CSF and may be useful for diagnosis and follow-up of patients with CNS tumours. Several markers have been reported to be elevated in the CSF of patients with PCNSL, such as IL-10, soluble IL-2 receptor (sIL-2R) and CXCL13, in comparison with patients with other types of CNS tumours or non-neoplastic conditions. $^{9798}$

Tryptophan is an essential amino acid implicated in tumour cell proliferation. A study using alpha- $\left[{ }^{11} \mathrm{C}\right]$ methyl-L-tryptophan PET in vivo showed increased uptake of tryptophan in gliomas, suggestive of increased tryptophan metabolism. ${ }^{99}$ Similarly, Locasale et al reported increased levels of tryptophan metabolites, such as indole, indoleacrylic acid and anthranilic acid, in the CSF of patients with recurrent gliomas. ${ }^{79} 83$ Histidine was also reported to be increased in the CSF of patients with brain tumours, along with other amino acids, such as valine, glutamine and asparagine. ${ }^{83} 100101$

Krivopusk found that the level of methionine was decreased in the CSF of patients with brain tumours. ${ }^{101}$ Methionine is an essential amino acid required for glioblastoma cell growth; in the absence of methionine, glioblastoma cells show 40\%-60\% reduction in cell proliferation. ${ }^{102}$ Palanichamy et al studied tryptophan and methionine and their respective metabolites, kynurenine and 5'-methylthioadenosine, in glioblastoma cell lines and normal human astrocytes. They found that levels of methionine, tryptophan and their metabolites were high in glioblastoma tumour cells, whereas in the extracellular space, methionine and tryptophan levels were low, and levels of their metabolites were increased. ${ }^{102}$

\section{Monoamine and polyamines}

Monoamine neurotransmitters include epinephrine, norepinephrine, serotonin and dopamine. They are derived from aromatic amino acids like phenylalanine, tyrosine and tryptophan. ${ }^{103}$ Monoamine neurotransmitters are involved in the regulation of cognitive processes such as emotion, arousal and certain types of memory. They also maintain neuronal integrity by causing the secretion of neurotrophin-3 from astrocytes. It has been reported that monoamines and their metabolites are increased in different brain tumours. The role of monogenic amines in the ventricular CSF of children suffering from posterior fossa tumours was studied by Varela et al. ${ }^{103}$ CSF was collected during placement of ventriculoperitoneal shunts and the levels of 3-methoxy-4-hydroxyphenylglycol (MHPG), homovanillic acid and 5-hydroxyindoleacetic acid were measured. They reported alterations in monoamine levels in the CSF from these patients and found that MHPG concentration in CSF was significantly higher in patients with astrocytomas than in patients with medulloblastomas.

Polyamines (eg, putrescine and spermidine) are another group of metabolites that are altered with brain tumours. They found 
Table 2 Summary of metabolites studied in CSF samples of patients with CNS tumours

\begin{tabular}{|c|c|c|c|c|}
\hline Tumour & Metabolites & Level & Cellular role & Reference \\
\hline \multicolumn{5}{|l|}{ Primary tumours } \\
\hline \multirow[t]{3}{*}{ Gliomas } & Citrate, isocitrate, pyruvate & $\uparrow$ & Glycolysis and TCA cycle & 7591100 \\
\hline & Oxaloacetate, malate, fumarate & $\downarrow$ & TCA cycle & 8191 \\
\hline & $\begin{array}{l}\text { Tryptophan, its metabolites, eg, indole, indoleacrylic acid, } \\
\text { anthranilic acid } \\
\text { Enolase } \\
\text { Myo-inositol }\end{array}$ & $\uparrow$ & $\begin{array}{l}\text { Inflammation } \\
\text { Enzyme in glycolysis } \\
\text { Cell signalling and cell differentiation }\end{array}$ & $\begin{array}{l}8399 \\
108 \\
8395\end{array}$ \\
\hline \multirow{11}{*}{$\begin{array}{l}\text { Wide range of brain } \\
\text { tumours, including } \\
\text { glioblastoma }\end{array}$} & Glucose & $\downarrow$ & Source of cellular energy & $\begin{array}{l}89909596 \\
109\end{array}$ \\
\hline & Lactate & $\uparrow$ & Product of pyruvate by LDH & $818486-88$ \\
\hline & Lactate dehydrogenase & $\uparrow$ & Enzyme involved in conversion of pyruvate to lactate & 8185 \\
\hline & Myo-inositol & Variable & Cell signalling and cell differentiation & 8395 \\
\hline & Cholesteryl sulfate & $\uparrow$ & Cell signalling & 8283 \\
\hline & $\begin{array}{l}\text { Various amino acids (eg, histidine, glutamine, asparagine, } \\
\text { valine) }\end{array}$ & $\uparrow$ & Amino acid metabolism & $100-102$ \\
\hline & Methionine & $\downarrow$ & & \\
\hline & Glutamine & Variable & Glycolysis, lipid, nucleic acid and protein synthesis & 95110 \\
\hline & Monoamine metabolites, eg, MHPG, HVA, 5-HIAA & Variable & Maintenance of neuronal integrity & $103111-113$ \\
\hline & Aspartate Aminotransferase (AST) & $\uparrow$ & Amino acid metabolism & 114 \\
\hline & Polyamine metabolites, eg, putrescine and spermidine & $\uparrow$ & Cell growth and cell proliferation & 104 \\
\hline Primary CNS lymphoma & Neopterin & $\uparrow$ & Inflammatory markers & 9798115 \\
\hline
\end{tabular}

CNS, central nervous system; CSF, cerebrospinal fluid; 5-HIAA, 5-hydroxyindoleacetic acid; HVA, homovanillic acid; LDH, lactate dehydrogenase; MHPG, 3-methoxy-4hydroxyphenylglycol; TCA, tricarboxylic acid.

to be correlated with cellular proliferation and growth in all eukaryotic cells. It has been demonstrated that polyamines might be useful biochemical markers for diagnosis and follow-up in patients with malignant neoplasms. Elevations in CSF concentrations of putrescine and spermidine were found in patients with untreated malignant CNS tumours compared with those observed in the CSF of patients without neoplasia. ${ }^{104}$

All the aforementioned metabolites have been found to be associated with primary CNS tumours. We summarise the metabolites discussed above and metabolites involved in metastatic CNS tumours in table 2.

\section{Obstacles in metabolomics}

Metabolomics can be challenging because it entails measurement of small molecules that vary in their physical properties. ${ }^{105} 106$ Preanalytical factors, diet and comorbidities could affect the levels of various metabolites in the CSF. These factors may be patient related such as differences in age, sex, genetics, lifestyle and dietary factors, or CSF-processing-related factors. The altered metabolites in CSF do not point towards a specific affected area of the brain. In addition, many metabolites associated with CNS tumours have been shown to be upregulated in serum or tumour tissue, but have not yet been measured in CSF. It also remains to be determined if levels of tumour-derived metabolites correlate with tumour burden and can be used to monitor response to treatment and tumour recurrence. Further studies are needed to identify critical molecules in the CSF that can aid in the diagnosis and monitoring of patients with CNS tumours.

\section{CONCLUSION}

In conclusion, collection and molecular analysis of CSF should be implemented in patients with CNS tumours. This strategy may lead to improved diagnosis, estimation of prognosis and therapeutic approaches for CNS tumours. This can be particularly helpful to avoid surgical intervention in high-risk patients or in those in which the tumour location is not amenable to resection. In addition, it could potentially allow monitoring of tumour response to treatment. However, major concerns remain regarding clinical applications of CSF biomarkers. Although there is a growing body of information on CSF biomarkers, it must be recognised that most studies have been performed by a small number of research groups and on limited numbers of samples. Studies employed different technologies and methods, and there is no optimised standard protocol for CSF collection, preparation, biomarker isolation and data analysis. Conclusive results cannot clearly be gathered for further clinical validation. A multi-institutional, longitudinal, collaborative study of a large cohort of patients is needed. ${ }^{9}$ Moreover, more comprehensive studies are required to specifically examine the impact of tumour type and tumour location in CNS in order to establish the reliability of different markers for each individual type of CNS tumour.

\section{Take home messages}

- Current methods for diagnosing and monitoring CNS tumors, such as CSF-cytology and MRI, have low sensitivity and specificity, respectively.

- Studies show that blood is not an ideal fluid to accurately evaluate biomarkers derived from CNS tumors, likely due to the presence of the blood brain barrier.

- Circulating biomarkers in CSF, including circulating tumor DNA, microRNAs, and metabolites, hold promise for accurate assessment of CNS tumors.

- CSF biomarkers may be helpful for preoperative diagnosis, diagnosis of tumors that are not amenable or typically treated by surgery, early identification of tumor recurrence, and monitoring treatment response. 
Handling editor Tahir S Pillay.

Contributors SZ, FI, MR, YE, PPA and LYB: literature review and manuscript writing.

Funding The authors have not declared a specific grant for this research from any funding agency in the public, commercial or not-for-profit sectors.

Competing interests None declared.

Patient consent Not required.

Provenance and peer review Not commissioned; externally peer reviewed.

\section{REFERENCES}

1 Siegel RL, Miller KD, Jemal A. Cancer statistics, 2016. CA Cancer J Clin 2016:66:7-30.

2 Hudson MM, Meyer WH, Pui CH. Progress born from a legacy of collaboration. J Clin Oncol 2015:33:2935-7.

3 Van Mieghem E, Wozniak A, Geussens Y, et al. Defining pseudoprogression in glioblastoma multiforme. Eur J Neurol 2013;20:1335-41.

4 Weston CL, Glantz MJ, Connor JR. Detection of cancer cells in the cerebrospinal fluid: current methods and future directions. Fluids Barriers CNS 2011;8:14

5 Bach F, Bjerregaard B, Sölétormos G, et al. Diagnostic value of cerebrospinal fluid cytology in comparison with tumor marker activity in central nervous system metastases secondary to breast cancer. Cancer 1993;72:2376-82.

6 Patel AS, Allen JE, Dicker DT, et al. Identification and enumeration of circulating tumor cells in the cerebrospinal fluid of breast cancer patients with central nervous system metastases. Oncotarget 2011;2:752-60.

7 Grossman SA, Krabak MJ, carcinomatosis L. Leptomeningeal carcinomatosis. Cancer Treat Rev 1999;25:103-19.

8 Glantz MJ, Cole BF, Glantz LK, et al. Cerebrospinal fluid cytology in patients with cancer. Cancer 1998;82:733-9.

9 Shalaby T, Achini F, Grotzer MA. Targeting cerebrospinal fluid for discovery of brain cancer biomarkers. J Cancer Metastasis Treat 2016;2:176.

10 Chamberlain MC, Glantz M, Groves MD, et al. Diagnostic tools for neoplastic meningitis: detecting disease, identifying patient risk, and determining benefit of treatment. Semin Oncol 2009;36-S35-S45.

11 Omuro A. Glioblastoma and other malignant gliomas. JAMA 2013;310:1842-50.

12 Haber DA, Gray NS, Baselga J. The evolving war on cancer. Cell 2011;145:19-24.

13 Gerlinger M, Rowan AJ, Horswell S, et al. Intratumor heterogeneity and branched evolution revealed by multiregion sequencing. N Eng/ J Med 2012;366:883-92.

14 Sottoriva A, Spiteri I, Piccirillo SG, et al. Intratumor heterogeneity in human glioblastoma reflects cancer evolutionary dynamics. Proc Natl Acad Sci U SA 2013;110:4009-14.

15 Diaz LA, Bardelli A. Liquid biopsies: genotyping circulating tumor DNA. J Clin Oncol 2014:32:579-86.

16 Heitzer E, Ulz P, Geigl JB. Circulating tumor DNA as a liquid biopsy for cancer. Clin Chem 2015:61:112-23.

17 Siravegna G, Mussolin B, Buscarino M, et al. Clonal evolution and resistance to EGFR blockade in the blood of colorectal cancer patients. Nat Med 2015;21:827-7.

18 Dawson S-J, Tsui DWY, Murtaza M, et al. Analysis of circulating tumor DNA to monitor metastatic breast cancer. $N$ Engl J Med Overseas Ed 2013:368:1199-209.

19 Murtaza M, Dawson SJ, Tsui DW, et al. Non-invasive analysis of acquired resistance to cancer therapy by sequencing of plasma DNA. Nature 2013:497:108-12.

20 Romanel A, Gasi Tandefelt D, Conteduca V, et al. Plasma AR and abirateroneresistant prostate cancer. Sci Trans/ Med 2015:7:re10.

21 Oxnard GR, Paweletz CP, Kuang Y, et al. Noninvasive detection of response and resistance in EGFR-mutant lung cancer using quantitative next-generation genotyping of cell-free plasma DNA. Clin Cancer Res 2014;20:1698-705.

22 Bettegowda C, Sausen M, Leary R, et al. Abstract 5606: Detection of circulating tumor DNA in early and late stage human malignancies. Cancer Res 2014;74-5606-6.

23 Pan W, Gu W, Nagpal S, et al. Brain tumor mutations detected in cerebral spinal fluid. Clin Chem 2015;61:514-22.

24 Müller C, Holtschmidt J, Auer M, et al. Hematogenous dissemination of glioblastoma multiforme. Sci Trans/ Med 2014;6:247ra101-1.

25 Macarthur KM, Kao GD, Chandrasekaran S, et al. Detection of brain tumor cells in the peripheral blood by a telomerase promoter-based assay. Cancer Res 2014:74:2152-9.

26 Sullivan JP, Nahed BV, Madden MW, et al. Brain tumor cells in circulation are enriched for mesenchymal gene expression. Cancer Discov 2014;4:1299-309.

27 De Mattos-Arruda L, Mayor R, Ng CK, et al. Cerebrospinal fluid-derived circulating tumour DNA better represents the genomic alterations of brain tumours than plasma. Nat Commun 2015;6:8839.

28 Bartlett F, Kortmann R, Saran F. Medulloblastoma. Clin Oncol 2013;25:36-45.

29 von Hoff K, Rutkowski S, Hoff von K. Medulloblastoma. Curr Treat Options Neurol $2012 ; 14: 416-26$
30 Deisenhammer F, Bartos A, Egg R. Routine cerebrospinal fluid (CSF) analysis. In: European handbook of neurological management. Oxford, UK: Wiley-Blackwell, 2010: 5-17.

31 Rhodes CH, Honsinger C, Sorenson GD. Detection of tumor-derived DNA in cerebrospinal fluid. J Neuropathol Exp Neurol 1994;53:364-8.

32 Rhodes $\mathrm{CH}$, Honsinger C, Sorenson GD. PCR-detection of tumor-derived p53 DNA in cerebrospinal fluid. Am J Clin Pathol 1995;103:404-8.

33 Park I, Bok R, Ozawa T, et al. Detection of early response to temozolomide treatment in brain tumors using hyperpolarized 13C MR metabolic imaging. J Magn Reson Imaging 2011;33:1284-90.

34 Shao H, Chung J, Balaj L, et al. Protein typing of circulating microvesicles allows realtime monitoring of glioblastoma therapy. Nat Med 2012;18:1835-40.

35 Li M, Diehl F, Dressman D, et al. BEAMing up for detection and quantification of rare sequence variants. Nat Methods 2006;3:95-7.

36 Shendure J, Ji H. Next-generation DNA sequencing. Nat Biotechnol 2008;26:1135-45

37 Wang $Y$, Springer $S$, Zhang $M$, et al. Detection of tumor-derived DNA in cerebrospinal fluid of patients with primary tumors of the brain and spinal cord. Proc Nat/ Acad Sci U SA 2015:112:9704-9.

38 Pentsova El, Shah RH, Tang J, et al. Evaluating cancer of the central nervous system through next-generation sequencing of cerebrospinal fluid. J Clin Oncol 2016:34:2404-15.

39 Ballester LY, Glitza Oliva IC, Douse DY, et al. Evaluating circulating tumor DNA from the cerebrospinal fluid of patients with melanoma and leptomeningeal disease. J Neuropathol Exp Neurol 2018;77:628-35.

40 Mitchell SM, Ross JP, Drew HR, et al. A panel of genes methylated with high frequency in colorectal cancer. BMC Cancer 2014;14:54.

41 Kurian AW, Hare EE, Mills MA, et al. Clinical evaluation of a multiplegene sequencing panel for hereditary cancer risk assessment. J Clin Oncol 2014;32:2001-9.

42 Glass JP, Melamed M, Chernik NL, et al. Malignant cells in cerebrospinal fluid (CSF): the meaning of a positive CSF cytology. Neurology 1979;29:1369-75.

43 Rhodes CH, Glantz MJ, Glantz L, et al. A comparison of polymerase chain reaction examination of cerebrospinal fluid and conventional cytology in the diagnosis of lymphomatous meningitis. Cancer 1996;77:543-8.

44 Shingyoji M, Kageyama $H$, Sakaida T, et al. Detection of epithelial growth factor receptor mutations in cerebrospinal fluid from patients with lung adenocarcinoma suspected of neoplastic meningitis. J Thorac Oncol 2011:6:1215-20.

45 Swinkels DW, de Kok JB, Hanselaar A, et al. Early detection of leptomeningeal metastasis by PCR examination of tumor-derived K-ras DNA in cerebrospinal fluid. Clin Chem 2000;46:132-3.

46 Li Y, Pan W, Connolly ID, et al. Tumor DNA in cerebral spinal fluid reflects clinical course in a patient with melanoma leptomeningeal brain metastases. J Neurooncol 2016;128:93-100

47 Zorofchian S, Lu G, Zhu J-J, et al. Detection of the MYD88 p.L265P mutation in the CSF of a patient with secondary central nervous system lymphoma. Front Oncol 2018:8:7151.

48 Hiemcke-Jiwa LS, Minnema MC, Radersma-van Loon JH, et al. The use of droplet digital PCR in liquid biopsies: a highly sensitive technique for MYD88 p.(L265P) detection in cerebrospinal fluid. Hematol Oncol 2018;36:E7.

49 Connolly ID, Li Y, Pan W, et al. A pilot study on the use of cerebrospinal fluid cell-free DNA in intramedullary spinal ependymoma. J Neurooncol 2017;135:29-36.

50 De Mattos-Arruda L. Liquid biopsy for HER2-positive breast cancer brain metastasis: the role of the cerebrospinal fluid. ESMO Open 2017:2:e000270.

51 Baraniskin A, Kuhnhenn J, Schlegel U, et al. Identification of microRNAs in the cerebrospinal fluid as biomarker for the diagnosis of glioma. Neuro Oncol 2012;14:29-33.

52 Gallego JA, Gordon ML, Claycomb K, et al. In vivo microRNA detection and quantitation in cerebrospinal fluid. J Mol Neurosci 2012;47:243-8

53 Teplyuk NM, Mollenhauer B, Gabriely G, et al. MicroRNAs in cerebrospinal fluid identify glioblastoma and metastatic brain cancers and reflect disease activity. Neuro Oncol 2012:14:689-700.

54 Verheul C, Kleijn A, Lamfers MLM. Cerebrospinal fluid biomarkers of malignancies located in the central nervous system. Handb Clin Neurol 2017:146:139-69.

55 Shi R, Wang PY, Li XY, et al. Exosomal levels of miRNA-21 from cerebrospinal fluids associated with poor prognosis and tumor recurrence of glioma patients. Oncotarget 2015:6:26971-81.

56 Drusco A, Bottoni A, Laganà A, et al. A differentially expressed set of microRNAs in cerebro-spinal fluid (CSF) can diagnose CNS malignancies. Oncotarget 2015;6:20829-39.

57 Lee Y, El Andaloussi S, Wood MJ. Exosomes and microvesicles: extracellular vesicles for genetic information transfer and gene therapy. Hum Mol Genet 2012;21-R125-R134.

58 Taylor DD, Gercel-Taylor C. MicroRNA signatures of tumor-derived exosomes as diagnostic biomarkers of ovarian cancer. Gynecol Oncol 2008:110:13-21.

59 Turchinovich A, Weiz L, Langheinz A, et al. Characterization of extracellular circulating microRNA. Nucleic Acids Res 2011;39:7223-33. 
60 Akers JC, Hua W, Li H, et al. A cerebrospinal fluid microRNA signature as biomarker for glioblastoma. Oncotarget 2017;8:68769-79.

61 Baraniskin A, Kuhnhenn J, Schlegel U, et al. MicroRNAs in cerebrospinal fluid as biomarker for disease course monitoring in primary central nervous system lymphoma. J Neurooncol 2012;109:239-44.

62 Xia H, Qi Y, Ng SS, et al. MicroRNA-15b regulates cell cycle progression by targeting cyclins in glioma cells. Biochem Biophys Res Commun 2009;380:205-10.

63 Le MT, Xie H, Zhou B, et al. MicroRNA-125b promotes neuronal differentiation in human cells by repressing multiple targets. Mol Cell Biol 2009;29:5290-305.

64 Le MT, Teh C, Shyh-Chang N, et al. MicroRNA-125b is a novel negative regulator of p53. Genes Dev 2009;23:862-76.

65 Xia HF, He TZ, Liu CM, et al. MiR-125b expression affects the proliferation and apoptosis of human glioma cells by targeting Bmf. Cell Physiol Biochem 2009;23-347-58.

66 Jin Z, Xu S, Yu H, et al. miR-125b inhibits Connexin43 and promotes glioma growth. Cell Mol Neurobiol 2013;33:1143-8.

67 Smits M, Wurdinger T, van het Hof B, et al. Myc-associated zinc finger protein (MAZ) is regulated by miR-125b and mediates VEGF-induced angiogenesis in glioblastoma. Faseb J 2012;26:2639-47.

68 Shi L, Zhang J, Pan T, et al. MiR-125b is critical for the suppression of human U251 glioma stem cell proliferation. Brain Res 2010;1312:120-6.

69 Spratlin JL, Serkova NJ, Eckhardt SG. Clinical applications of metabolomics in oncology: a review. Clin Cancer Res 2009;15:431-40.

70 Pandey R, Caflisch L, Lodi A, et al. Metabolomic signature of brain cancer. Mol Carcinog 2017:56:2355-71.

71 Markley JL, Brüschweiler R, Edison AS, et al. The future of NMR-based metabolomics. Curr Opin Biotechnol 2017;43:34-40.

72 Dudley E, Yousef M, Wang Y. Targeted metabolomics and mass spectrometry. Elsevier, 2010: 45-83.

73 Dettmer K, Aronov PA, Hammock BD. Mass spectrometry-based metabolomics. Mass Spectrom Rev 2007;26:51-78.

74 Griffin JL, Shockcor JP. Metabolic profiles of cancer cells. Nat Rev Cancer 2004:4:551-61.

75 Vander Heiden MG, Cantley LC, Thompson CB. Understanding the Warburg effect: the metabolic requirements of cell proliferation. Science 2009;324:1029-33.

76 Ishikawa S, Sugimoto M, Kitabatake K, et al. Identification of salivary metabolomic biomarkers for oral cancer screening. Sci Rep 2016:6:31520.

77 Tiziani S, Kang Y, Harjanto R, et al. Metabolomics of the tumor microenvironment in pediatric acute lymphoblastic leukemia. PLoS One 2013;8:e82859.

78 Zhang T, Wu X, Ke C, et al. Identification of potential biomarkers for ovarian cancer by urinary metabolomic profiling. J Proteome Res 2013;12:505-12.

79 Wang H, Tso VK, Slupsky CM, et al. Metabolomics and detection of colorectal cancer in humans: a systematic review. Future Oncol 2010;6:1395-406.

80 Lin L, Huang Z, Gao Y, et al. LC-MS based serum metabonomic analysis for renal cell carcinoma diagnosis, staging, and biomarker discovery. J Proteome Res 2011;10:1396-405.

81 Nakamizo S, Sasayama T, Shinohara M, et al. GC/MS-based metabolomic analysis of cerebrospinal fluid (CSF) from glioma patients. J Neurooncol 2013;113:65-74.

82 Wishart DS, Feunang YD, Marcu A, et al. HMDB 4.0: the human metabolome database for 2018. Nucleic Acids Res 2018;46-D608-D617.

83 Locasale JW, Melman T, Song S, et al. Metabolomics of human cerebrospinal fluid identifies signatures of malignant glioma. Mol Cell Proteomics 2012;11:M111.014688

84 Tennant DA, Durán RV, Boulahbel H, et al. Metabolic transformation in cancer. Carcinogenesis 2009;30:1269-80.

85 Balmaceda C, Gaynor JJ, Sun M, et al. Leptomeningeal tumor in primary central nervous system lymphoma: recognition, significance, and implications. Ann Neurol 1995;38:202-9.

86 Komorowski RA, Farmer SG, Hanson GA, et al. Cerebrospinal fluid lactic acid in diagnosis of meningitis. J Clin Microbio/ 1978;8:89-92.

87 Chow SL, Rooney ZJ, Cleary MA, et al. The significance of elevated CSF lactate. Arch Dis Child 2005;90:1188-9.

88 Blüher S, Schulz M, Bierbach U, et al. Central lactic acidosis, hyperventilation, and respiratory alkalosis: leading clinical features in a 3-year-old boy with malignant meningeal melanoma. Eur J Pediatr 2008;167:483-5.

89 Laszlo MH, LEVINE MA. Low cerebrospinal-fluid sugar content in meningeal and intracranial neoplasia. JAMA 1965;193:834-5.
90 Erdem G, Topçu M, Topaloğlu H, et al. Dermoid tumor with persistently low CSF glucose and unusual CT and MRI findings. Pediatr Neurol 1994;10:75-7.

91 Reitman ZJ, Jin G, Karoly ED, et al. Profiling the effects of isocitrate dehydrogenase 1 and 2 mutations on the cellular metabolome. Proc Natl Acad Sci U SA 2011;108:3270-5.

92 Yan H, Parsons DW, Jin G, et al. IDH1 and IDH2 mutations in gliomas. N Engl J Med 2009;360:765-73.

93 Dang L, White DW, Gross S, et al. Cancer-associated IDH1 mutations produce 2-hydroxyglutarate. Nature 2010;465:966-6.

94 Kalinina J, Ahn J, Devi NS, et al. Selective detection of the d-enantiomer of 2-hydroxyglutarate in the CSF of glioma patients with mutated isocitrate dehydrogenase. Clin Cancer Res 2016;22:6256-65.

95 Kallenberg K, Bock HC, Helms G, et al. Untreated glioblastoma multiforme: increased myo-inositol and glutamine levels in the contralateral cerebral hemisphere at proton MR spectroscopy. Radiology 2009;253:805-12.

96 Castillo M, Smith JK, Kwock L. Correlation of myo-inositol levels and grading of cerebral astrocytomas. AJNR Am J Neuroradiol 2000;21:1645-9.

97 Sasayama T, Nakamizo S, Nishihara M, et al. Cerebrospinal fluid interleukin-10 is a potentially useful biomarker in immunocompetent primary central nervous system lymphoma (PCNSL). Neuro Oncol 2012;14:368-80.

98 Rubenstein JL, Wong VS, Kadoch C, et al. CXCL13 plus interleukin 10 is highly specific for the diagnosis of CNS lymphoma. Blood 2013;121:4740-8.

99 Juhász C, Chugani DC, Muzik O, et al. In vivo uptake and metabolism of alpha[11C]methyl-L-tryptophan in human brain tumors. J Cereb Blood Flow Metab 2006;26:345-57.

100 Koschorek F, Gremmel H, Stelten J, et al. Characterization of CNS lesions by using high-resolution $1 \mathrm{H}$ MR spectroscopy of CSF: preliminary results. AJNR Am J Neuroradiol 1989:10:523-7.

101 Krivopusk ME. [Amino acid composition of ventricular and lumbar cerebrospinal fluid in brain tumors]. Zh Nevropatol Psikhiatr Im S S Korsakova 1977;77:813-4.

102 Palanichamy K, Thirumoorthy K, Kanji S, et al. Methionine and kynurenine activate oncogenic kinases in glioblastoma, and methionine deprivation compromises proliferation. Clin Cancer Res 2016;22:3513-23.

103 Varela M, Alexiou GA, Liakopoulou M, et al. Monoamine metabolites in ventricular CSF of children with posterior fossa tumors: correlation with tumor histology and cognitive functioning. J Neurosurg Pediatr 2014;13:375-9.

104 Marton LJ, Heby 0, Levin VA, et al. The relationship of polyamines in cerebrospinal fluid to the presence of central nervous system tumors. Cancer Res 1976;36:973-7.

105 Rosenling T, Slim CL, Christin C, et al. The effect of preanalytical factors on stability of the proteome and selected metabolites in cerebrospinal fluid (CSF). J Proteome Res 2009;8:5511-22.

106 Kuehnbaum NL, Britz-McKibbin P. New advances in separation science for metabolomics: resolving chemical diversity in a post-genomic era. Chem Rev 2013:113:2437-68.

107 Baraniskin A, Kuhnhenn J, Schlegel U, et al. Identification of microRNAs in the cerebrospinal fluid as marker for primary diffuse large B-cell lymphoma of the central nervous system. Blood 2011;117:3140-6.

108 Royds JA, Timperley WR, Taylor CB. Levels of enolase and other enzymes in the cerebrospinal fluid as indices of pathological change. J Neurol Neurosurg Psychiatry 1981:44:1129-35.

109 Chow E, Troy SB. The differential diagnosis of hypoglycorrhachia in adult patients. Am J Med Sci 2014;348:186-90.

110 el-Yazigi A, Al-Mefty 0. Decreased cerebrospinal fluid glutamine levels in patients with benign brain tumors. J Neurochem 1985;45:815-8.

111 Porta M, Bareggi SR, Collice M, et al. Determination of HVA and 5-HIAA in ventricular CSF of patients with brain tumors. J Neurosurg Sci 1974;18:157-63.

112 Bareggi SR, Porta M, Collice M, et al. Monoamine acid metabolites in ventricular CSF of patients with brain tumours. Acta Neurochir 1976;35-161-70.

113 Bostrom B, Mirkin BL. Elevation of cerebrospinal fluid catecholamine metabolites in patients with intracranial tumors of neuroectodermal origin. J Clin Oncol 1987;5:1090-7.

114 Dharker SR, Dharker RS, Chaurasia BD. Lactate dehydrogenase and aspartete transaminase of the cerebrospinal fluid in patients with brain tumours, congenital hydrocephalus, and brain abscess. J Neurol Neurosurg Psychiatry 1976;39:1081-5.

115 Viaccoz A, Ducray F, Tholance Y, et al. CSF neopterin level as a diagnostic marker in primary central nervous system lymphoma. Neuro Oncol 2015;17:1497-503. 